\title{
Rational Design of Gold Nanostars with Tailorable Plasmonic Properties
}

\author{
Ted V. Tsoulos, ${ }^{1}$ Supriya Atta, ${ }^{2}$ Maureen J. Lagos, ${ }^{1,3}$ Philip E. Batson, ${ }^{1,3}$ George Tsilomelekis, ${ }^{4}$ \\ and Laura Fabris*1
}

${ }^{1}$ Department of Materials Science and Engineering, Rutgers University, 607 Taylor Road Piscataway NJ 08854.

${ }^{2}$ Department of Chemistry and Chemical Biology, Rutgers University, 610 Taylor Road Piscataway NJ 08854.

${ }^{3}$ Department of Physics \& Astronomy, Rutgers University, 136 Frelinghuysen Road, Piscataway NJ 08854.

${ }^{4}$ Department of Chemical and Biochemical Engineering, Rutgers University, 98 Brett Road, Piscataway NJ 08854.

KEYWORDS: Plasmons, Nanostars, Colloidal Synthesis, Nano-Antennas, EELS

\begin{abstract}
Gold nanostars display exceptional field enhancement properties and tunable resonant modes that can be leveraged to create effective imaging tags or phototherapeutic agents, or to design novel hot-electron based photocatalysts. From a fundamental standpoint, they represent important tunable platforms to study the dependence of hot carrier energy and dynamics on plasmon band intensity and position. Toward the realization of these platforms, holistic approaches taking into account both theory and experiments to study the fundamental behavior of these
\end{abstract}


particles are needed. Arguably, the intrinsic difficulties underlying this goal stem from the inability to rationally design and effectively synthesize nanoparticles that are sufficiently monodispersed to be employed for corroborations of the theoretical results without the need of single particle experiments. Herein, we report on our concerted computational and experimental effort to design, synthesize, and explain the origin and morphology-dependence of the plasmon modes of a novel gold nanostar system, with an approach that builds upon the well-known plasmon hybridization model. We have synthesized monodispersed samples of gold nanostars with finely tunable morphology employing seed-mediated colloidal protocols, and experimentally observed narrow and spectrally resolved harmonics of the primary surface plasmon resonance mode both at the single particle level (via electron energy loss spectroscopy) and in ensemble (by UV-Vis and ATRFTIR spectroscopies). Computational results on complex anisotropic gold nanostructures are validated experimentally on samples prepared colloidally, underscoring their importance as ideal testbeds for the study of structure-property relationships in colloidal nanostructures of high structural complexity.

\section{MAIN TEXT}

Gold nanostars have shown to possess plasmonic properties with the potential to enable technological breakthroughs in various fields, such as imaging ${ }^{1}$, sensing $^{2}$, and catalysis ${ }^{3}$. Importantly, their limited cytotoxicity ${ }^{4}$ promises to enable their use in vitro and in vivo. However, despite the wealth of applied work ${ }^{5,6,7}$, very limited fundamental research has been carried out on these particles, both from the experimental and theoretical standpoints ${ }^{8,9,10}$. As a result, published research cannot be evaluated comparatively, and, therefore, implemented at the industrial scale for technologically relevant applications. Thus, in an effort to rationally take advantage of the outstanding field enhancement properties of these particles, there is an important need to carry out 
fundamental-level research that holistically hinges on both computational predictions and experimental verifications.

In 2007, Nordlander and coworkers proposed a theoretical framework to describe the localized surface plasmon resonances (LSPR) of gold nanostars ${ }^{11}$. In this model, known as the plasmon hybridization model ${ }^{12}$, they suggested that the LSPR bands observed in the visible-near infrared (vis-NIR) can be described as hybrid resonances of those pertaining to the spherical core and the individual protruding spikes, leading to bright and dark modes. They also showed that the position of the multipolar modes depends on the length and sharpness of the spikes, and their relative orientation with respect to the polarization of the incoming field, with longer and sharper spikes displaying more red-shifted resonances ${ }^{11}$. Since these seminal discoveries however, very limited theoretical work has appeared that focused on gold nanostars, despite the substantial ongoing experimental efforts.

Arguably, one of the reasons hampering the push for fundamental understanding is the lack of synthetic protocols that ensure sufficient monodispersity and batch-to-batch reproducibility. This limited sample monodispersity leads to substantially broadened LSPR bands that cannot be used to unequivocally correlate morphology features to relevant plasmon modes. As a consequence, experimental results have not been able to fully support the theoretical endeavors. Gold nanorods and nanowires have been successfully utilized for many years to study localized and propagating plasmons as well as to demonstrate the concept of wave-particle duality ${ }^{13}$. Despite the unquestioned importance of these studies and the impressive improvements in the synthetic protocols to achieve monodispersed nanorod with high tunability ${ }^{14}$, including post-synthetic manipulations ${ }^{15}$, the $1 \mathrm{D}$ morphology of nanorods and nanowires does not provide sufficient degrees of freedom for the generation and observation of unique patterns of plasmon interactions, 
such as those possible when spherical cores couple to sharp spikes protruding at various relative orientations. They, therefore, represent ideal cases of a more general 3D model. Hence, the necessity of developing novel protocols for the rational synthesis of anisotropic plasmonic nanostructures remains an unmet need and, still, a long standing goal. Over the years, scientists have developed various methods for the synthesis of these particles, both seeded and non seedmediated, either employing surfactants or $\operatorname{not}^{16,17}$. So far however, even though substantial improvements have been reported in several protocols ${ }^{18}, 19$, reproducibility and monodispersity do not meet the necessary quality levels.

In this work we report on a combined theoretical and experimental effort to provide an indepth fundamental understanding of the plasmonic properties of gold nanostars, which attempts to extend the framework developed through the plasmon hybridization model to a more generalized system. We show how we were able to successfully establish a causal relationship between the morphology of the particles and their measured plasmonic behavior. Theoretically, we demonstrate the presence of an intense fundamental mode in the short wave infrared (SWIR) regime and its higher harmonics at higher energy. Synthetically, we report a new protocol that displays extraordinary monodispersity and reproducibility. The unbiased agreement between experimental and computational results, both at the single-particle level and in ensemble, proves the validity of our model and confirms the monodispersity of our synthetic approach. We believe that this work will provide a much needed impulse for the implementation of these nanostars in technologicallyrelevant applications and possibly for the development of scale-up strategies relevant to industriallevel synthesis. 


\section{RESULTS AND DISCUSSION}

By employing 3D finite element simulations via the Comsol Multiphysics software, we have implemented a theoretical framework to study the plasmonic properties of gold nanostars in which core and spikes are considered as inseparable units, all contributing to the number, position, and intensity of the resulting LSPR modes (see Methods section for details). Our method, while verifying and reproducing the results by Nordlander ${ }^{11}$ for nanostars characterized by numerous short spikes protruding from the spherical core (typically obtained employing surfactant-free seeded protocols), departs partially from it. More specifically, it has evidenced additional plasmonic properties in nanostars characterized by few, long, high aspect ratio spikes, such as the onset and disappearance of resonances when the spikes interact at angles other than 180 degrees. Our frequency domain simulations also indicate that bulk plasmons appear to be generated as image charges inside the spikes owing to their limited thickness. Interestingly, our model has shown that the core appears to affect the position of the resonant modes, thus acting as an electron reservoir and interfering, as a consequence, with the charge distribution along the spikes. This prediction has been confirmed via single particle electron energy loss (EELS) experiments. By modeling nanostars with spike lengths increasing between 70 and $100 \mathrm{~nm}$, we have observed an intense resonance band at wavelengths longer than $1000 \mathrm{~nm}$, that can be assigned to the first harmonic of the propagating interface charge waves. Higher order harmonics (i.e. second and third) can be identified that resonate between 600 and $1000 \mathrm{~nm}$, with spacing between the three that depends on the slant length of the conical spike. Importantly, our model predicts a linear dependence between the position of the harmonic modes and the spike length, a correlation that was confirmed experimentally via Vis-NIR-SWIR spectrophotometry and ATR-FTIR spectroscopy. These results are promising in that they lay the foundations for the rational design 
of colloidal nanostructures with plasmonic properties that can be tailored for specific applications and reproduced synthetically with high monodispersity and reproducibility.
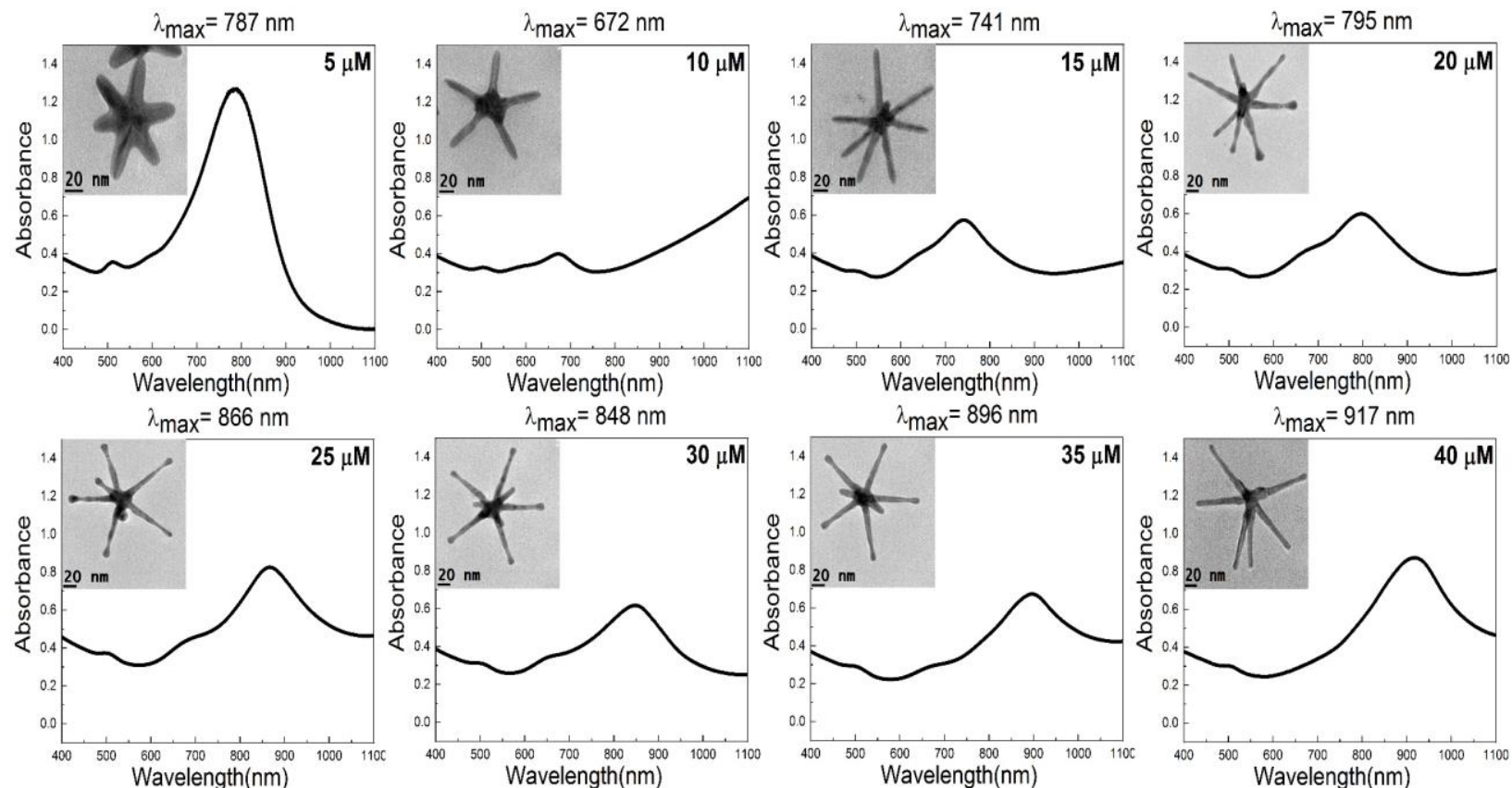

Figure 1. Transmission electron micrographs and UV-Vis spectra of gold nanostar samples obtained by increasing the amount of $\mathrm{AgNO}_{3}$ between 5 and $40 \mu \mathrm{M}$ as described in the Methods section. The resulting UV-Vis spectra are characterized by a prominent LSPR mode that shifts between $672 \mathrm{~nm}$ (at $10 \mu \mathrm{M} \mathrm{AgNO} 3$ concentration) and $917 \mathrm{~nm}$ (at $40 \mu \mathrm{M} \mathrm{AgNO} 3$ concentration) and can be described as the second harmonic of the primary dipolar mode, which resonates above $1100 \mathrm{~nm}$ and thus cannot be observed in these spectra. For the $5 \mu \mathrm{M} \mathrm{AgNO}_{3}$ concentration case, the observed prominent band is the first harmonic mode, which in this case appears at higher energies because of the shorter spike length obtained at this $\mathrm{AgNO}_{3}$ concentration. It is important to observe how the morphology of the nanostars, and of the spikes in particular, changes in close correlation to the amount of $\mathrm{AgNO}_{3}$ added. Scale bars at $20 \mathrm{~nm}$.

In order to determine the accuracy of the model and corroborate the theoretical predictions, we hypothesized that a synthetic protocol was needed that could provide nanostars with 1) a limited number of spikes, 2) tunable spike length, 3) high monodispersity, and 4) consistent batch-to-batch reproducibility. So far, such a detailed synthetic protocol ensuring that all these requirements would be met simultaneously has not been reported. Leveraging and optimizing a synthetic 
protocol in which the concerted action of Triton X, ascorbic acid, and silver nitrate leads to gold nanostars characterized by LSPR bands that can be tuned through the addition of increasing amounts of $\mathrm{AgNO}_{3}$ (see Figure 1) ${ }^{20}$, we have been able to synthesize highly monodispersed 6branched gold nanostar samples with enhanced batch-to-batch reproducibility and tunable spike morphology. In particular, the high sample monodispersity (vide infra), coupled to the tunability enabled by our synthesis, represent an excellent opportunity to employ these particles to corroborate the predictions of our model. The detailed synthetic procedures and growth mechanisms, including the dependence between the concentration of $\mathrm{AgNO}_{3}$ and the spike length are the focus of a more specialized manuscript soon to be published. The possibility to bridge the gap between theory and experiment with a truly 3D model that does not make constraining structural assumptions, promises to lead to a complete understanding of structure-plasmonic property relationships in these complex nanomaterials. We have investigated in depth the size and shape dependence of the plasmon resonances for the synthesized 6-branched nanostars, reporting in Figure 2 the results for particles with spike lengths between $70 \mathrm{~nm}$ and $100 \mathrm{~nm}$. Size and shape can be tuned by rationally varying the relative concentrations of $\mathrm{AgNO}_{3}$, ascorbic acid, Triton $\mathrm{X}$, and seeds, as well as reaction time reaction time (Figure S1). Furthermore, detailed dimensional analysis over samples larger than 100 particles revealed extraordinary monodispersity (both within and among batches), with maximum standard deviations of $\pm 4 \%$, as reported in the representative histograms in Figure 2 (i-1). Analysis of nanostar morphology has also shown that, although some of the nanostars possess five or seven spikes, the majority of them are characterized by six branches, hence the chosen nomenclature. More detailed TEM tomographic reconstruction is current undergoing to obtain additional details on this observation, including the correlation between the number of spikes and that of the twin defects present on the initial seeds. 

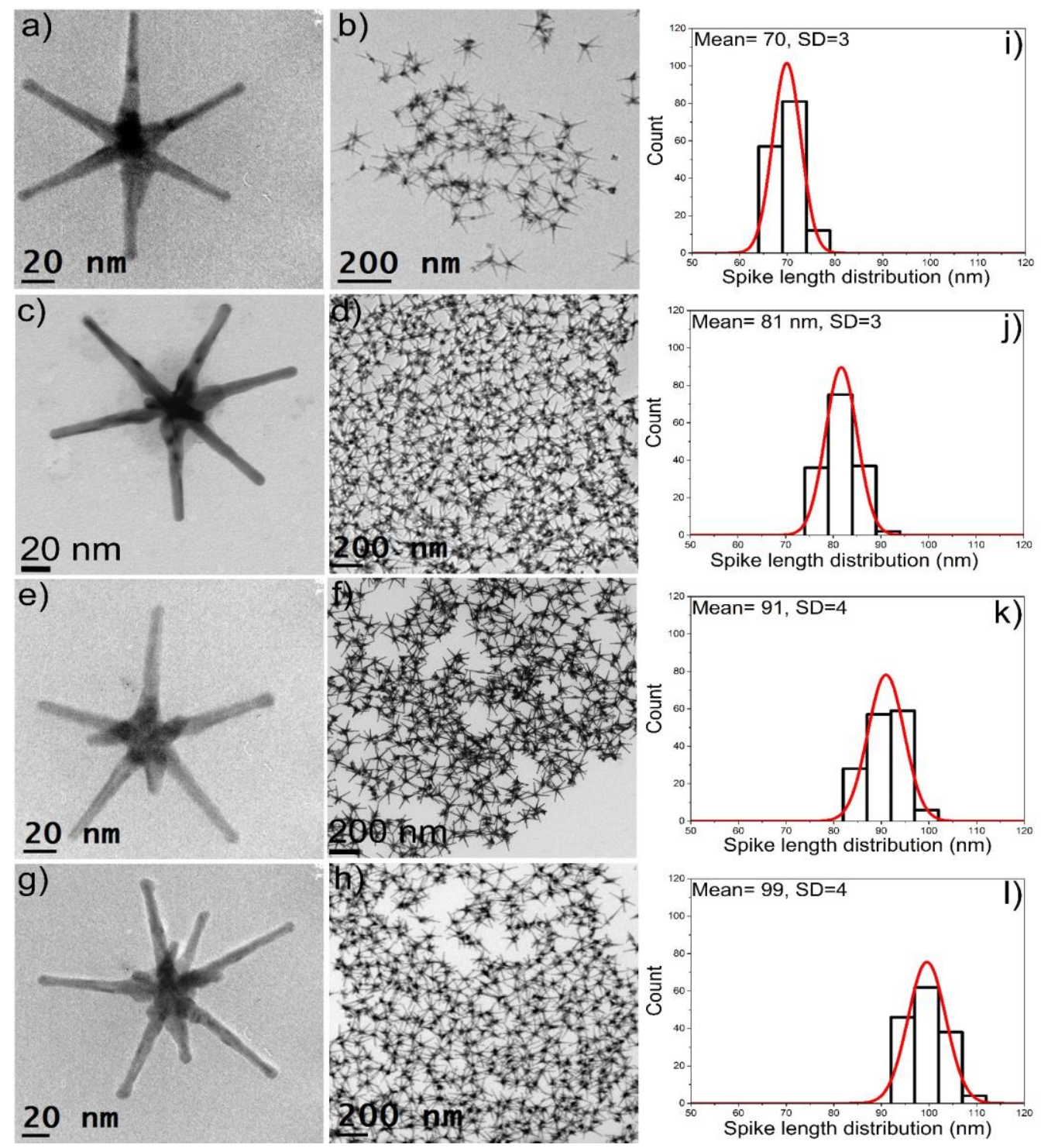

$20 \mathrm{~nm}$

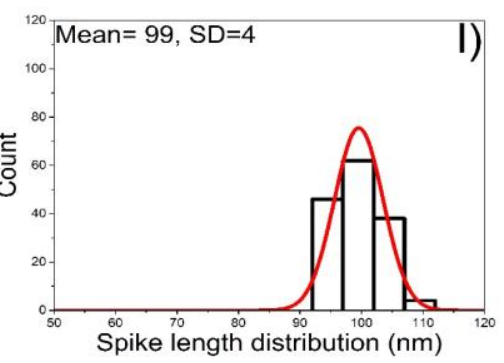

Figure 2. Representative transmission electron microscopy (TEM) micrographs of 6-branched gold nanostars in the order of increasing spike length (a, c, e, and g). These syntheses lead to monodispersed samples in high yield, as evidenced in the TEM micrographs (b, $\mathrm{d}, \mathrm{f}$, and $\mathrm{h}$ ). Statistical analysis of the spike length distribution of 6-branched gold nanostars was obtained by measuring the length of 150 spikes from different areas on the TEM grid for each sample. Spike length analysis: i) Mean: $70 \mathrm{~nm}$; Standard deviation: $3 \mathrm{~nm}$, j) Mean: $81 \mathrm{~nm}$; Standard deviation: $3 \mathrm{~nm}, \mathrm{k}$ ) Mean: $91 \mathrm{~nm}$; Standard deviation: $4 \mathrm{~nm}$, and 1) Mean: $99 \mathrm{~nm}$; Standard deviation: $4 \mathrm{~nm}$. 

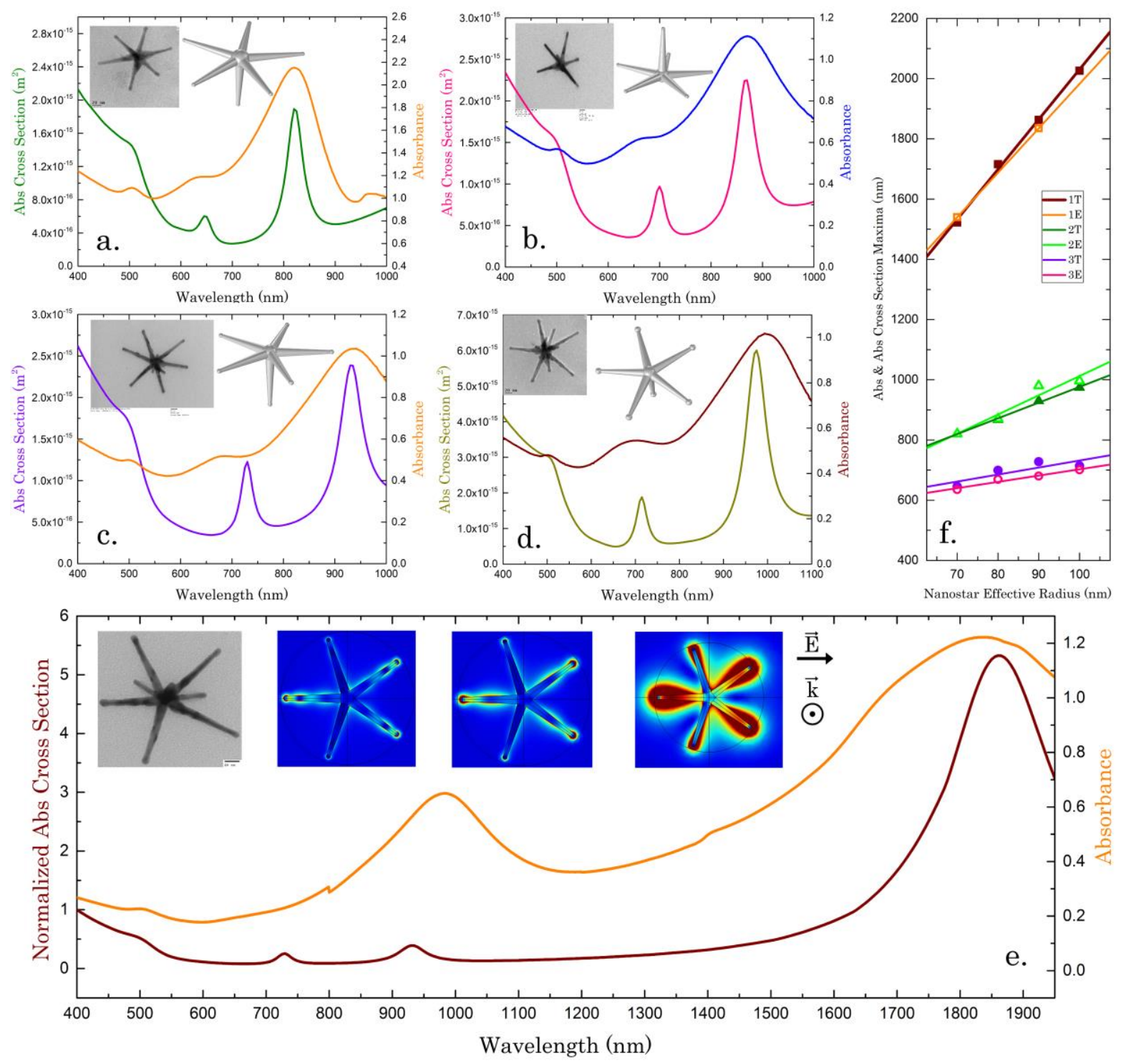

Figure 3. Experimental and theoretical spectra of 6-spike nanostars of a) 70, b) 80, c) 90 and d) $100 \mathrm{~nm}$ spike lengths. e) Long range theoretical and experimental spectra of $90 \mathrm{~nm}$ radius 6spike nanostars. E-field norm (calculated via Eq. 5 in Methods section) maps of the first, second, and third harmonic modes at $1865 \mathrm{~nm}, 930 \mathrm{~nm}$, and $730 \mathrm{~nm}$, respectively. Spectra normalization was carried out with respect to the photon energy of each wavelength compared to the photon energy at $400 \mathrm{~nm}$. FWHM for first and second harmonic are $242 \mathrm{~nm}$ and $65 \mathrm{~nm}$, respectively, for the calculated spectra, and $500 \mathrm{~nm}$ and $190 \mathrm{~nm}$, respectively, for the experimental ones. f) Linear dependence (actual fits) between the harmonic modes resonant maxima (1,2 and 3) and the nanostar spike lengths. Note the decreasing slope as we move towards higher harmonics and the very good matching between theoretical $(\mathrm{T})$ predictions and experimental (E) results. 
To deepen our understanding of the plasmon resonance-morphology relationship, we synthesized samples with spike lengths of $70 \mathrm{~nm}, 80 \mathrm{~nm}, 90 \mathrm{~nm}$, and $100 \mathrm{~nm}$ and compared the optical spectra collected spectrophotometrically in ensemble averaged experiments with the results of our calculations (Figure 3). We have compared the vis-NIR spectra with the absorption cross section spectra obtained through our model (Figure 3 a-d); in particular, the very good agreement between the position and normalized intensity of the bands modeled and measured (Figure 3e) confirms the validity of our model. The experimental validation, both at the single particle level and in ensemble, suggests that plasmonic resonances in gold nanostars should be interpreted as harmonics of the main LSPR mode. When nanostars with shorter and thicker spikes are studied, the higher harmonics, being less intense and more closely spaced compared to the case of nanostars with high aspect ratio spikes, seemingly disappear giving rise to the well-known broad LSPR bands observed for surfactant free gold nanostars ${ }^{21,22}$. In reality they become simply enveloped within the more intense fundamental mode. Inter-spike coupling, common in the case of multi-spike nanostars (again observed in surfactant free gold nanostar syntheses) also causes them to slightly blue shift, further broadening the LSPR band. Our study, proposing a more generalized approach to the interpretation of LSPR bands in gold nanostars, predicts very well the position of the harmonics based on the detailed morphology of the nanostar in exam. The core diameter, the length, and the sharpness of the spikes, along with the tip morphology (Figure S2), dictate the position and distance between the harmonics. The relative position of the bands governs the linear relationship reported in Figure 3f, and follows a simplified rule in agreement with antenna theory

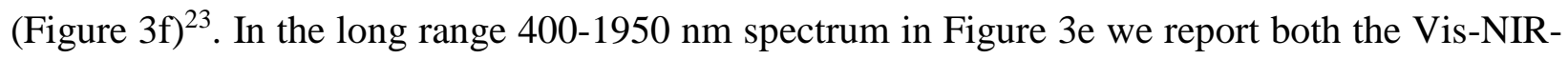
SWIR spectrum and the calculated normalized absorption cross section of a $90 \mathrm{~nm}$ nanostar, along with three E-field maps highlighting the spatial distribution of the field at the position of the 
maxima for the three observed harmonics. The very good agreement between position and intensity modeled and experimentally measured provides a solid experimental proof of the validity of our model, which is further supported by the FTIR and EELS results presented onwards. Interestingly, our model suggests that the first harmonic mode, in addition to displaying substantially higher intensity compared to its higher harmonics, appears to extend in space much farther from the surface than the other two, which could be leveraged in techniques such as surface enhanced Raman scattering (SERS) where E-field enhancements are lead to higher signal intensity and assay sensitivity.

In Figure 4, we present the ATR-FTIR spectra of highly concentrated aqueous solutions of gold nanostars with spike lengths spanning between $70 \mathrm{~nm}$ and $100 \mathrm{~nm}$. The full spectral range, $700-7500 \mathrm{~cm}^{-1}$, is reported in Figure S3. In the $4000-6500 \mathrm{~cm}^{-1}$ spectral range, we observe the peak maxima for the first harmonic to be centered at $\sim 5700 \mathrm{~cm}^{-1}, 5180 \mathrm{~cm}^{-1}, 4930 \mathrm{~cm}^{-1}$, and 4830 $\mathrm{cm}^{-1}$ for the $70,80,90$, and $100 \mathrm{~nm}$ respectively. The peak positions of the four different samples fit nicely the E-field norm introduced by the theorectical predictions and the Vis-NIR-SWIR spectroscopy measurements. A noticeable shift in the observed first harmonic peaks can be attributed to interparticle coupling effects (Figure S4), since the FTIR measurements took place in concentrated solutions as compared to the Vis-NIR spectrophotometric measurements.

Interestingly, the observed center of each peak appears to be linearly dependent on the average size of the gold nanostars in solution, demonstrating the strong dependence of this plasmonic mode on the length of the spike, as described in the previous section and plotted in Figure $4 \mathrm{~d}$ and $4 \mathrm{e}$. The results obtained from the ATR-FTIR spectra further underscore the ability and reliability of the developed protocol for the synthesis of colloidal nanostars with plasmonic 

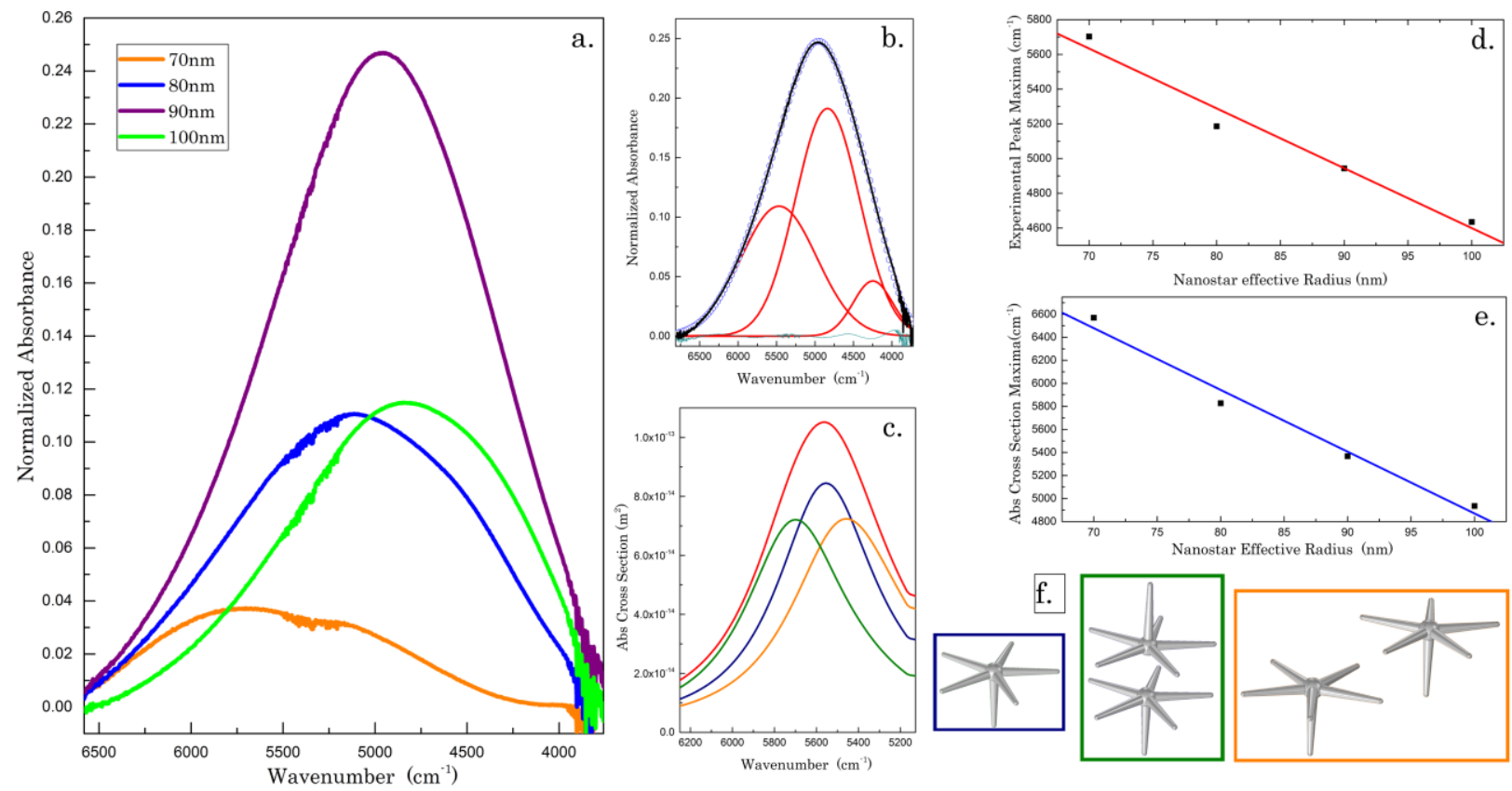

Figure 4. FTIR spectra collected on nanostars with $70 \mathrm{~nm}, 80 \mathrm{~nm}, 90 \mathrm{~nm}$, and $100 \mathrm{~nm}$ spike lengths. Normalization carried out with respect to the area under the curve of each spectrum (a). Spectrum of a suspension of nanostars with $90 \mathrm{~nm}$ spike length deconvoluted with Gauss function (b). Theoretical absorption cross section spectra of 1) a single $90 \mathrm{~nm}$ nanostar, 2) two nanostars with their in-plane spikes kept parallel at $50 \mathrm{~nm}$ distance, 3) two nanostars aligned tip-to-tip at 50 $\mathrm{nm}$, along with the average of these three spectra shown in red (c and f). Linear fitting of the observed FTIR peak maxima shows a strong interdependence between the first harmonic resonances and the spike length experimentally (d) and theoretically (e).

properties that can be rationally tailored for specific applications. It is worth mentioning here that contrary to the well defined, Gaussian-type predicted peaks by the model, in our ATR-FTIR spectra the first harmonic appears to be a convolution of more than one contributions in the overall spectral envelope. To address this, all the spectra were deconvoluted using Gauss functions based on the Levenberg-Marquardt algorithm as shown elsewhere ${ }^{24,25,26,27}$. The deconvoluted spectra show two or three major peaks separated by approximately $800 \mathrm{~cm}^{-1}$.

To further investigate the latter, in Figure $4 \mathrm{~b}$ and $4 \mathrm{c}$ we report the comparison between the deconvoluted ATR-FTIR spectrum and the calculated absorption cross section for $90 \mathrm{~nm}$-spike nanostars. The distinct peaks in ATR-FTIR can be attributed to the same plasmonic mode under 
different geometrical configurations (Figure 4f). Theoretical results (Figure 4c and 4f) show that changing the distance as well as the configuration between two adjacent nanostars can both blue shift and red shift the plasmonic mode. The theoretical treatment to nanostar coupling aims to outline the importance of considering multiple geometric configurations between randomly dispersed adjacent nanostars at high concentrations when interpreting the experimental results. We have investigated three of these numerous possible configurations and report the shifts of the first harmonic with respect to the distance between nanostars (Figure S4).

To further confirm the theoretical predictions, we performed EELS studies to evaluate the plasmonic response of the gold nanostars at the single particle level. While single particle scattering data could be in first principle proposed to assess the plasmonic response, the presence of the substrates, coupling anisotropically to the nanostars, would have further complicated the experimental response, and was therefore not deemed accurate to assess the LSPR bands in this particular study. Figure 5 exhibits EELS spectra acquired on a 6-spike nanostar with the electron beam probing the nanostructure in non-intersecting and intersecting geometries. Since the plasmon coupling between spikes is very weak due to their large physical separation ${ }^{28}$, one can in principle analyze their plasmonic behavior considering an isolated spike coupled to the star core. This approach allows us to probe a single spike and then repeat the process for the other spikes of the stars. We obtained the same findings for all of the spikes, as illustrated in Figure 5 (left side-spike 1; right side-spike 2). Note that the spectra can exhibit up to two resonances associated with the first $(670 \mathrm{meV})$ and second $(1360 \mathrm{meV})$ harmonic excitations, depending on the beam location. The resonance energies are similar to those found by optical techniques (UV-Vis-NIR and ATRFTIR, Figures 3 and 4 respectively) and match well the theoretical findings. When the electron beam is located in regions near/within the tip (position 1,3) the excitation of the first harmonic 
nanostructure can be predicted theoretically and observed experimentally owing to the extraordinary monodispersity and reproducibility of the synthetic protocols. While other nonspherical nanoparticles (e.g. nanorods) have been synthesized with high degree of monodispersity and synthetic control, the morphology of the particles shown herein is substantially more complex to achieve. In-depth multi-technique characterization has shown that the peak positions of the plasmonic resonances linearly depend on the length of the spikes, with slight variations originating from the specific tip- and spike-morphologies. The proposed theoretical model has predicted a strong plasmonic mode, along with its higher order eigenmodes, in excellent detail. EELS data, collected on isolated spikes, have confirmed the influence of the electrons of the core on the spectral position of the various harmonics; in addition, they have underscored the importance of taking into consideration the effect of the electron beam in intersecting probing geometries. From the single particle level to the highly interacting ensemble, the agreement between theory and experiment advocates for the need of a combined approach towards a rational and effective evolution of plasmonic materials and methods. The high monodispersity and batch-to-batch reproducibility of the samples, ensured by our synthetic protocol, suggest that these 6-branched nanostars might be ideal testbeds for the experimental validation of plasmonics theory. The model has also suggested the presence of bulk image charges within the tips, that couple with the surface charges owing to the reduced thickness of the spikes. In addition, it appears to indicate that propagating polaritons, traveling along the high aspect ratio nanostar spikes, might coexist and interact with the highly localized modes at the tips. Ongoing studies in our lab are further investigating these observations and will be the focus of future publications. 


\section{METHODS}

\section{Modeling}

Using a linearly polarized (LP) incident light source, as seen in Equation 1, with an electric field of amplitude $1 \mathrm{~V} / \mathrm{m}$, we simulated the optical properties of nanostars using a model developed in the RF Module of COMSOL Multiphysics 5.0.

1)

$$
\boldsymbol{E}=e^{-j \boldsymbol{k}_{0} z} \hat{x}
$$

The simulations were based on numerical solutions of a time-averaged Laplacian, seen in Equation 2, employing the widely used Finite Element Method.

$$
\nabla \times \frac{1}{\mu_{r}}(\nabla \times \boldsymbol{E})-k_{0}^{2}\left(\varepsilon_{r}-\frac{j \sigma}{\omega \varepsilon_{0}}\right) \boldsymbol{E}=\mathbf{0}
$$

The geometry was built on three concentric domains. A fully absorbing spherical layer, usually reffered to as perfectly matched layer (PML), a layer assigned the dielectric properties of water, from Hale and Querry ${ }^{30}$, and a core domain including the various nanostar models assigned the dielectric properties of gold from the Lorentz-Drude model by Johnson and Christy ${ }^{31}$. The relative permeability $\mu_{\mathrm{r}}$ was taken to be 1 in all geometry domains without loss of generality. The angular frequency $\omega$ was calculated from the input wavelength values.

The nanostar models were also developed in Comsol Multiphysics using mostly a spherical core, eccentric conical spikes with hemispherical, oblate, and prolate hemiellipsoidal tips. The 
volume and surface areas of these models are defined by Equations 3 and $\mathbf{4}$ with $i$ being the number of spikes, varying from 1 to 6 in this work.

$$
\begin{gathered}
V=\frac{4}{3} \pi a^{3}+\sum_{i=1}^{n}\left\{\frac{h_{i}}{3} \pi\left(R_{i}^{2}+R_{i} r_{i}+r_{i}^{2}\right)+\frac{2}{3} \pi r_{i}^{3}-\frac{\pi}{6}\left(a-\sqrt{a^{2}-R_{i}^{2}}\right)\left[3 R_{i}^{2}+\right.\right. \\
\left.\left.\left(a-\sqrt{a^{2}-R_{i}^{2}}\right)^{2}\right]\right\} \\
A=4 \pi a^{2}-\sum_{i=1}^{n}\left\{2 \pi a\left(a-\sqrt{a^{2}-R_{i}^{2}}\right\}+\sum_{i=1}^{n}\left\{\pi\left(r_{i}+R_{i}\right) \sqrt{\left(R_{i}-r_{i}\right)^{2}+h_{i}^{2}}+2 \pi r_{i}^{2}\right\}\right.
\end{gathered}
$$

Furthermore, $a$ is the radius of the spherical core, $R$ is the base radius of a spike, $r$ is the radius of the hemispherical tip of a spike, and $h$ is the distance between $R$ and $r$. Note that these equations describe only the cases of nanostars with hemispherical tips.

The various nanostar models were built to match the shapes and complexity of nanostars synthesized experimentally, and characterized via transmission electron microscopy (TEM), as described below. Herein, for sake of completeness, we compared the results collected via simulations to both those obtained on individual nanostars via electron energy loss spectroscopy (EELS) and to the ensemble averaged ones derived from the large-number statistical analysis of nanostar suspensions. Importantly, the excellent sample monodispersity and batch-to-batch reproducibility achievable with the synthetic protocol described herein, allow us, for the first time, to exactly match the plasmonic resonances observed experimentally to those calculated computationally, thus enabling us to identify new resonant and coupling modes in these nanoparticles.

The layered geometry was meshed employing randomly distributed tetrahedra, whose size was kept between 0.1 and $2 \mathrm{~nm}$ within the NS domain and at less than $4 \mathrm{~nm}$ in the surrounding 
medium domain. Numerical solutions of Equation 2 were accomplished through an iterative method in which the difference between individual solutions in the constituent tetrahedral elements is minimized within a tolerance of $10^{-6}$. The whole procedure is based on convergence of the individual solutions within the above tolerance.

From the solution of Equation 2, we provide plots of the normalized scattered electric field. Three dimensional plots of the electric field norm (Equation 5) on the surface of the various nanostars along with two dimensional slice plots of the electric field norm are presented. 2D slice plots are fundamental to study, at the same time, surface and bulk plasmon modes in these nanoparticles.

$$
|\boldsymbol{E}|=\sqrt{E_{x}^{2}+E_{y}^{2}+E_{z}^{2}}
$$

In parallel, we calculate the absorption, scattering, and extinction cross sections, as defined by Equations 6 and 7.

$$
\sigma_{a b s}=\frac{\boldsymbol{W}_{a b s}}{\boldsymbol{P}_{\text {inc }}} \quad \sigma_{s c a}=\frac{\boldsymbol{W}_{s c a}}{\boldsymbol{P}_{\text {inc }}}
$$

$$
\sigma_{e x t}=\sigma_{a b s}+\sigma_{s c a}
$$

The absorption and scattering cross sections are defined by the energy rates absorbed and scattered by the nanostar volume and surface area, defined in Equations 8 and 9 respectively, and divided by the incident irradiance, given by Equation 10: 


$$
\left.\boldsymbol{W}_{a b s}=\frac{1}{2} \iiint_{\mathrm{xyz}}\left[\operatorname{Re}\left[(\sigma \mathbf{E}+j \omega \boldsymbol{D}) \cdot \mathbf{E}^{*}\right]+j \omega \boldsymbol{B}\right) \cdot \mathbf{H}^{*}\right] \mathrm{dxdydz}(\mathrm{W})
$$

$$
\begin{gathered}
\boldsymbol{W}_{s c a}=\oiint\left[\boldsymbol{E} \times \boldsymbol{H}^{*}\right] \cdot \mathbf{n d S}(\mathrm{W}) \\
\boldsymbol{P}_{\text {inc }}=\frac{1}{2 Z_{0}}\left|\boldsymbol{E}_{0}\right|^{2}
\end{gathered}
$$

where $Z_{0}$ is the impedance of free space and $E_{0}$ is the amplitude of the incident electric field, 1 $\mathrm{V} / \mathrm{m}$ as seen in Equation 1.

Additionally, given the extinction cross section, we calculate the shape- and concentrationdependent extinction coefficient by multiplying the extinction cross section with the number density of nanostars as seen in Equation 11:

$$
\varepsilon_{\text {dep }}=\sigma_{\text {ext }} n
$$

Similarly, the absorption coefficient is given by Equation 12.

$$
a=\sigma_{a b s} n
$$

This is a concentration dependent quantity, since it contains the number density of nanostars n. In this work, it is compared to the experimental absorption coefficient, obtained from the measured absorbance from Equation 15. We deduce Equation 15 by combining Equation 13, the BeerLambert law and the definition of absorption coefficient Equation 14, where Abs is the 
experimentally measured absorbance, $\mathrm{I}$ and $\mathrm{I}_{0}$ the incident and transmitted light intensities, $\mathrm{z}$ the light path length through the cuvette holding the aqueous NS suspensions, and $\alpha_{\exp }$ the experimental absorption coefficient.

$$
\begin{gathered}
A b s=\log _{10} \frac{I_{0}}{I} \\
I=I_{0} e^{-\alpha_{\text {exp }} z} \\
\alpha_{\text {exp }}=\frac{\ln (10) A b s}{z}
\end{gathered}
$$

The relationships described above allow us to directly compare the calculated absorption cross section spectra to the UV-Vis-NIR absorption spectra obtained experimentally, as in detail described in our previous work ${ }^{32}$.

\section{Instrumentation.}

UV-Vis-NIR Spectrophotometry. Absorption spectra were recorded on a Thermo Scientific Evolution $300 \mathrm{UV}-\mathrm{V}$ is spectrophotometer using a quartz cuvette with $1 \mathrm{~cm}$ path. A Cary Series UV-Vis-NIR Spectrophotometer (Cary 5000 UV-Vis-NIR) was used to collect the UV-Vis-NIR spectra. $1 \mathrm{~mm}$ quartz cuvette was used with double beam mode to get the UV-Vis-NIR spectrum. 
Transmission Electron Microscopy. Nanoparticle morphology was determined using a Topcon 002B transmission electron microscope depositing the nanoparticle suspension on Ted Pella Inc. PELCO TEM grids.

ATR-FTIR Spectroscopy. A Thermo Fisher iS50 FTIR spectrometer equipped with a deuterated, L-alanine-doped triglycine sulfate (DLaTGS) detector, and a Golden Gate single-reflection diamond ATR was used for all spectroscopic studies. The instrument was equipped with a purge gas of dehumidified air (Parker-Balston 75-45) to remove water vapor. The resolution of the instrument was set at $4 \mathrm{~cm}^{-1}$ for the whole set of measurements and the number of scans varied from 16 to 32 . For each measurement, $\sim 100 \mu \mathrm{ml}$ of the gold nanostar aqueous solution were placed on the ATR crystal and left there for 5 mins to settle. The spectra were collected at $25^{\circ} \mathrm{C}$ by using air as reference background.

Electron Energy Loss Spectroscopy. Experimental conditions of high-beam current probes were used, typically attained with a condenser-lens setting, favoring higher beam current over spatial resolution. The spatial resolution was kept between $1.5 \AA$ and $2.0 \AA$. The EELS spectrometer entrance aperture subtends a 20 -mrad $\left(2.5 \AA^{-1}\right)$ half-angle at $60 \mathrm{kV}$. The EELS spectra were acquired in the aloof and intersecting geometries over $500 \mathrm{~ms}$ and 1s, respectively. A custom-made monochromator was employed to achieve $60 \mathrm{meV}$ energy resolution. Plasma cleaning and mild heat treatment at $75^{\circ} \mathrm{C}$ for six hours were employed to pretreat the sample to eliminate the surfactant layer and avoid carbon contamination under the electron beam. 


\section{Nanostar Synthesis}

Materials. Gold (III) chloride trihydrate $\left(\mathrm{HAuCl}_{4} \bullet 3 \mathrm{H}_{2} \mathrm{O}\right)$, silver nitrate $\left(\mathrm{AgNO}_{3} ; 99.995 \%\right), \mathrm{L}(+)$ ascorbic acid, sodium borohydride $\left(\mathrm{NaBH}_{4}\right)$, and TritonX-100 were purchased from SigmaAldrich. All these chemicals were used without further purification. Ultrapure MilliQ water (18.2 $\mathrm{M} \Omega \bullet \mathrm{cm}$ ) was used in all syntheses. All glassware was aqua regia cleaned before each synthesis.

Synthesis of 6 Branched Gold Nanostars. 6-branched gold nanostars were synthesized following a modified seed-mediated method first developed by Taglietti and coworkers ${ }^{20}$. Briefly, the seed solution was prepared by adding $0.6 \mathrm{ml}$ of a fresh, ice-cold $0.01 \mathrm{M} \mathrm{NaBH}_{4}$ solution to a mixture containing $0.1 \mathrm{ml}$ of $25 \mathrm{mM} \mathrm{HAuCl}_{4}$ and $10 \mathrm{ml}$ of $0.15 \mathrm{M}$ Triton X. After addition of $\mathrm{NaBH}_{4}$ the solution turned immediately from pale yellow to orange-red. The mixture was stirred for 2 minutes, and aged for 10 minutes at $4{ }^{\circ} \mathrm{C}$ before use. The growth solution was prepared by adding $40 \mu \mathrm{L}$ of $0.788 \mathrm{M}$ ascorbic acid to a solution containing $0.4 \mathrm{ml}$ of $25 \mathrm{mM} \mathrm{HAuCl}_{4}$, and $20 \mathrm{ml}$ of $0.15 \mathrm{M}$ of Triton-X. A variable amount of $\mathrm{AgNO}_{3}$ solution was added to the growth solution to obtain a

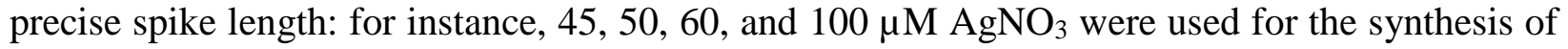
the $80,70,90$, and $100 \mathrm{~nm}$ spike length respectively. After that, a variable amount of seeds were added to the growth solution. Specifically, nanostars with $70 \mathrm{~nm}$ spike length were synthesized by addition of $24 \mu \mathrm{L}$ (14 $\mu \mathrm{L}$ for the other three spike lengths) seed solutions to the growth solution. The color of the solution turned from colorless to green after addition of the seeds, with the color becoming more intense after 5 minutes. The solution was gently stirred at room temperature for 12 hours. The conical spike morphology with spherical tip was obtained when $25 \mu \mathrm{M} \mathrm{AgNO}_{3}$ were added to the growth solution, and the reaction time was 10 minutes. The rod morphology was obtained when $15 \mu \mathrm{M} \mathrm{AgNO}_{3}$ was added to the growth solution, and the reaction time was 10 
minutes. The solution was purified via centrifugation at 4,000 $\mathrm{g}$ for $10 \mathrm{~min}$ and redispersed with 5 $\mathrm{ml}$ of Ultrapure MilliQ water (18.2 $\mathrm{M} \Omega \bullet \mathrm{cm})$.

Spike Length Measurement of Gold Nanoparticles. The core diameter, spike length, and aspect ratio of nanostars were measured by ImageJ software. The spike length was measured from the center of the core to the tip of the spike. We have measured 150 spikes for each different type nanostar to generate the reported statistics.

\section{ASSOCIATED CONTENT}

\section{Supporting Information}

Additional details of the 6-spike nanostars synthesis' developed protocols, modelling results and analytical description of the coupling between nanostars under three different geometric configurations, a graph and discussion on the monodispersity of the synthesized colloid by juxtaposition between the measured absorption spectrum and the calculated absorption cross section spectra of slightly different models and full range ATR-FTIR spectra.

\section{AUTHOR INFORMATION}

Corresponding Author

*E-mail: $\underline{\text { lfabris@soe.rutgers.edu }}$

Phone: +1-848-445-5606

\section{REFERENCES}

1. Rotz MW, Culver KSB, Parigi G, MacRenaris KW, Luchinat C, Odom TW, et al. High Relaxivity Gd(III)-DNA Gold Nanostars: Investigation of Shape Effects on Proton Relaxation. ACS Nano 2015, 9(3): 3385-3396. 
2. Indrasekara ASDS, Meyers S, Shubeita S, Feldman LC, Gustafsson T, Fabris L. Gold nanostar substrates for SERS-based chemical sensing in the femtomolar regime. Nanoscale 2014, 6(15): 8891-8899.

3. Sousa-Castillo A, Comesaña-Hermo M, Rodríguez-González B, Pérez-Lorenzo M, Wang Z, Kong X$\mathrm{T}$, et al. Boosting Hot Electron-Driven Photocatalysis through Anisotropic Plasmonic Nanoparticles with Hot Spots in Au-TiO2 Nanoarchitectures. The Journal of Physical Chemistry C 2016, 120(21): 11690-11699.

4. Bhamidipati M, Fabris L. Multiparametric Assessment of Gold Nanoparticle Cytotoxicity in Cancerous and Healthy Cells: The Role of Size, Shape, and Surface Chemistry. Bioconjugate Chemistry 2017, 28(2): 449-460.

5. Liu Y, Yuan H, Fales A, Register J, Vo-Dinh T. Multifunctional gold nanostars for molecular imaging and cancer therapy. Frontiers in Chemistry 2015, 3(51).

6. Ginting RT, Kaur S, Lim D-K, Kim J-M, Lee JH, Lee SH, et al. Plasmonic Effect of Gold Nanostars in Highly Efficient Organic and Perovskite Solar Cells. ACS Applied Materials \& Interfaces 2017, 9(41): 36111-36118.

7. Munkhbat B, Ziegler J, Pöhl H, Wörister C, Sivun D, Scharber MC, et al. Hybrid Multilayered Plasmonic Nanostars for Coherent Random Lasing. The Journal of Physical Chemistry C 2016, 120(41): 23707-23715.

8. Hrelescu C, Sau TK, Rogach AL, Jäckel F, Feldmann J. Single gold nanostars enhance Raman scattering. Applied Physics Letters 2009, 94(15): 153113.

9. Shao L, Susha AS, Cheung LS, Sau TK, Rogach AL, Wang J. Plasmonic Properties of Single Multispiked Gold Nanostars: Correlating Modeling with Experiments. Langmuir 2012, 28(24): 8979-8984.

10. Hrelescu C, Sau TK, Rogach AL, Jäckel F, Laurent G, Douillard L, et al. Selective Excitation of Individual Plasmonic Hotspots at the Tips of Single Gold Nanostars. Nano Letters 2011, 11(2): 402407.

11. Hao F, Nehl CL, Hafner JH, Nordlander P. Plasmon Resonances of a Gold Nanostar. Nano Letters 2007, 7(3): 729-732.

12. Prodan E, Radloff C, Halas NJ, Nordlander P. A Hybridization Model for the Plasmon Response of Complex Nanostructures. Science 2003, 302(5644): 419. 
13. Piazza L, Lummen TTA, Quiñonez E, Murooka Y, Reed BW, Barwick B, et al. Simultaneous observation of the quantization and the interference pattern of a plasmonic near-field. Nature Communications 2015, 6: 6407.

14. Ortiz N, Zoellner B, Hong SJ, Ji Y, Wang T, Liu Y, et al. Harnessing Hot Electrons from Near IR Light for Hydrogen Production Using Pt-End-Capped-AuNRs. ACS Applied Materials \& Interfaces 2017, 9(31): 25962-25969.

15. González-Rubio G, Díaz-Núñez P, Rivera A, Prada A, Tardajos G, González-Izquierdo J, et al. Femtosecond laser reshaping yields gold nanorods with ultranarrow surface plasmon resonances. Science 2017, 358(6363): 640-644.

16. Wu H-L, Chen C-H, Huang MH. Seed-Mediated Synthesis of Branched Gold Nanocrystals Derived from the Side Growth of Pentagonal Bipyramids and the Formation of Gold Nanostars. Chemistry of Materials 2009, 21(1): 110-114.

17. Grzelczak M, Perez-Juste J, Mulvaney P, Liz-Marzan LM. Shape control in gold nanoparticle synthesis. Chemical Society Reviews 2008, 37(9): 1783-1791.

18. Yuan H, Khoury CG, Hwang H, Wilson CM, Grant GA, Vo-Dinh T. Gold nanostars: surfactant-free synthesis, 3D modelling, and two-photon photoluminescence imaging. Nanotechnology 2012, 23(7): 075102.

19. Atta S, Tsoulos TV, Fabris L. Shaping Gold Nanostar Electric Fields for Surface-Enhanced Raman Spectroscopy Enhancement via Silica Coating and Selective Etching. The Journal of Physical Chemistry C 2016, 120(37): 20749-20758.

20. Pallavicini P, Dona A, Casu A, Chirico G, Collini M, Dacarro G, et al. Triton X-100 for three-plasmon gold nanostars with two photothermally active NIR (near IR) and SWIR (short-wavelength IR) channels. Chemical Communications 2013, 49(56): 6265-6267.

21. Hsiangkuo Y, Christopher GK, Hanjun H, Christy MW, Gerald AG, Tuan V-D. Gold nanostars: surfactant-free synthesis, 3D modelling, and two-photon photoluminescence imaging. Nanotechnology 2012, 23(7): 075102.

22. Chirea M, Collins SSE, Wei X, Mulvaney P. Spectroelectrochemistry of Silver Deposition on Single Gold Nanocrystals. The Journal of Physical Chemistry Letters 2014, 5(24): 4331-4335.

23. Paolo B, Jer-Shing H, Bert H. Nanoantennas for visible and infrared radiation. Reports on Progress in Physics 2012, 75(2): 024402. 
24. Fischer D, Pötschke $P$, Brünig $H$, Janke A. Investigation of the Orientation in Composite Fibers of Polycarbonate with Multiwalled Carbon Nanotubes by Raman Microscopy. Macromolecular Symposia 2005, 230(1): 167-172.

25. Kalampounias AG, Tsilomelekis G, Boghosian S. Unraveling the role of microenvironment and hydrodynamic forces on the vibrational relaxation rates of pyridine-water complexes. Journal of Molecular Liquids 2014, 198(Supplement C): 299-306.

26. Kalampounias AG, Tsilomelekis G, Boghosian S. Glass-forming ability of $\mathrm{TeO}(2)$ and temperature induced changes on the structure of the glassy, supercooled, and molten states. J Chem Phys 2015, 142(15): 154503.

27. Pötschke $P$, Brünig $H$, Janke $A$, Fischer $D$, Jehnichen $D$. Orientation of multiwalled carbon nanotubes in composites with polycarbonate by melt spinning. Polymer 2005, 46(23): 1035510363.

28. Mazzucco S, Geuquet N, Ye J, Stéphan O, Van Roy W, Van Dorpe P, et al. Ultralocal Modification of Surface Plasmons Properties in Silver Nanocubes. Nano Letters 2012, 12(3): 1288-1294.

29. Bosman M, Ye E, Tan SF, Nijhuis CA, Yang JKW, Marty R, et al. Surface Plasmon Damping Quantified with an Electron Nanoprobe. Scientific Reports 2013, 3: 1312.

30. Hale GM, Querry MR. Optical Constants of Water in the 200-nm to 200- $\mu \mathrm{m}$ Wavelength Region. Appl Opt 1973, 12(3): 555-563.

31. Johnson PB, Christy RW. Optical Constants of the Noble Metals. Physical Review B 1972, 6(12): 4370-4379.

32. Tsoulos TV, Han L, Weir J, Xin HL, Fabris L. A closer look at the physical and optical properties of gold nanostars: an experimental and computational study. Nanoscale 2017, 9(11): 3766-3773. 
TABLE OF CONTENT FIGURE for "Rational Design of Gold Nanostars with Tailorable Plasmonic Properties", by Ted V. Tsoulos, Supriya Atta, Maureen J. Lagos, Philip E. Batson, George Tsilomelekis, and Laura Fabris.

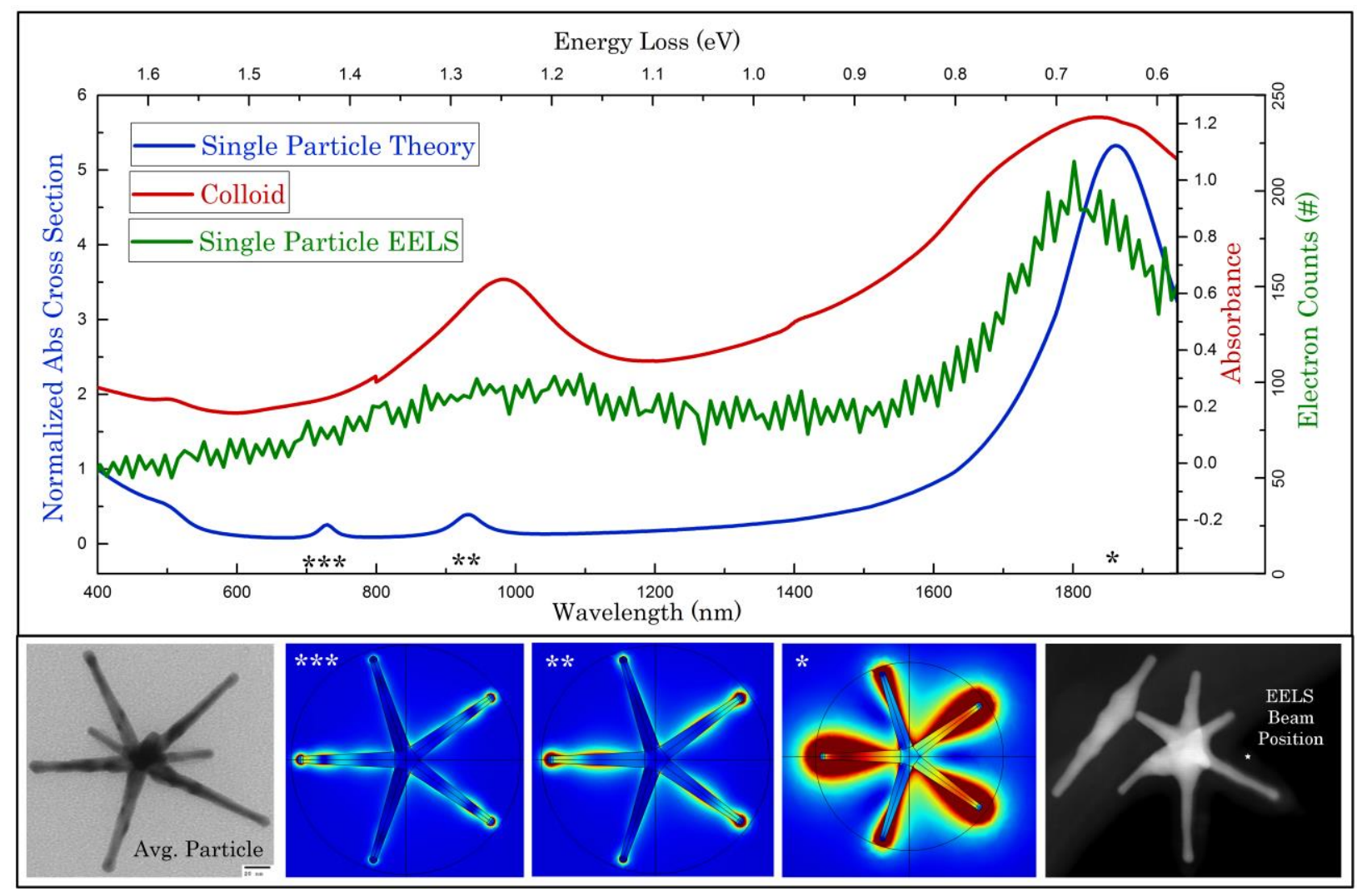

